\title{
Comparing machine learning algorithms for predicting COVID-19 mortality
}

\author{
Khadijeh Moulaei ${ }^{1}$, Mostafa Shanbehzadeh² ${ }^{2}$ Zahra Mohammadi-Taghiabad ${ }^{3}$ and Hadi Kazemi-Arpanahi ${ }^{4,5^{*}}$
}

\begin{abstract}
Background: The coronavirus disease (COVID-19) hospitalized patients are always at risk of death. Machine learning (ML) algorithms can be used as a potential solution for predicting mortality in COVID-19 hospitalized patients. So, our study aimed to compare several ML algorithms to predict the COVID-19 mortality using the patient's data at the first time of admission and choose the best performing algorithm as a predictive tool for decision-making.

Methods: In this study, after feature selection, based on the confirmed predictors, information about 1500 eligible patients (1386 survivors and 144 deaths) obtained from the registry of Ayatollah Taleghani Hospital, Abadan city, Iran, was extracted. Afterwards, several ML algorithms were trained to predict COVID-19 mortality. Finally, to assess the models' performance, the metrics derived from the confusion matrix were calculated.

Results: The study participants were 1500 patients; the number of men was found to be higher than that of women (836 vs. 664) and the median age was 57.25 years old (interquartile 18-100). After performing the feature selection, out of 38 features, dyspnea, ICU admission, and oxygen therapy were found as the top three predictors. Smoking, alanine aminotransferase, and platelet count were found to be the three lowest predictors of COVID-19 mortality. Experimental results demonstrated that random forest (RF) had better performance than other ML algorithms with accuracy, sensitivity, precision, specificity, and receiver operating characteristic (ROC) of 95.03\%, 90.70\%, 94.23\%, $95.10 \%$, and $99.02 \%$, respectively.
\end{abstract}

Conclusion: It was found that ML enables a reasonable level of accuracy in predicting the COVID-19 mortality. Therefore, ML-based predictive models, particularly the RF algorithm, potentially facilitate identifying the patients who are at high risk of mortality and inform proper interventions by the clinicians.

Keywords: COVID-19, Coronavirus, Machine learning, Artificial intelligence, Prediction hospital mortality

\section{Background}

In December 2019, the novel coronavirus disease (COVID-19) was detected in Wuhan District [1], Republic of China (ROC). Ever since, this virus has rapidly spread all over the world. In January 2020, World Health Organization (WHO) declared this outbreak as a pandemic $[2,3]$. The clinical outcomes of the virus ranged from asymptomatic or mild symptoms to serious

\footnotetext{
*Correspondence: h.kazemi@abadanums.ac.ir

${ }^{4}$ Department of Health Information Technology, Abadan University

of Medical Sciences, Abadan, Iran

Full list of author information is available at the end of the article
}

complications and, consequently, death in some cases. COVID-19 is a highly contagious viral infection and, thus far, continues to spread aggressively worldwide and has become a serious global health concern. Rapid spread of COVID-19 has resulted in the severe shortage of medical resources and exhaustion of frontline healthcare workers [4-9]. Moreover, many COVID-19 patients exacerbate rapidly after a period of quite mild symptoms, stressing the call for advanced risk stratification models. Applying predictive models can identify patients who are at the increased risk of mortality and provide support to reduce deaths as soon as possible [10-15]. Hence, to mitigate the burden on the healthcare system and provide the 
best care for patients, it is necessary to predict the prognosis of the disease and effectively triage critically the ill patients. Besides, due to the great hesitation surrounding its concluding influence, clinicians and health policymakers have commonly used and depended upon predictions made by different computational and statistical models $[16,17]$.

In response to the above-mentioned challenges, healthcare systems across the world attempt to leverage machine learning (ML) classifiers for achieving proper decision-making via eliminating physicians' subjective evaluations $[11,18,19]$. ML as a branch of artificial intelligence $(\mathrm{AI})$ enables extracting high-quality predictive models from the mining of huge raw datasets [20]. It is a valuable tool that is even more employed in medical research to improve predictive modeling and reveal new contributing factors of a specific target outcome [20, 21]. ML algorithms can reduce uncertainty and ambiguity by offering evidence-based medicine for risk analysis, screening, prediction, and care plans; they support reliable clinical decision-making and hope to improve patient outcomes and quality of care [22,23].

This study aimed to develop a mortality risk prediction model for COVID-19 based on ML algorithms that utilize patients' routine clinical data. We are mostly looking for the following questions: (1) What are the most relevant predictors of mortality among COVID-19 inhospital patients? (2) What is the best ML algorithm for developing the mortality prediction model?

\section{Methods}

\section{Feature identification and patient selection}

This stage contained the identification of the proposed features in predicting mortalities in the patients with COVID-19. At the first step, the most relevant clinical features were determined using an extensive literature review in scientific databases. Then, a questionnaire was designed through derived features (predictors) that belonged to the patient's demographics, risk factors, clinical manifestation, laboratory tests, and therapeutic classes. The content validity of the questionnaire was assessed by an expert panel including two infectious diseases specialists and two virologists. In addition, a test-retest (at 10-day interval) was done to evaluate the reliability of the questionnaire. Finally, the proposed clinical features were validated using a two-round Delphi survey by a group of multidisciplinary expert team, including five infectious diseases specialists, three epidemiologists, and two virologists. The experts were asked to review the initial list of the parameters to score each item according to their importance in predicting COVID-19 mortality based on a 5-point Likert scale, ranging from 1 to 5 , where 1 indicates "not important" and 5 indicates "highly important". Only the features with the average score of 3.75 (70\%) and higher were allowed in the study.

After performing a literature review coupled with a two-round Delphi survey, based on finalized feature set, data from laboratory-confirmed COVID-19 hospitalized patients $(n=1500)$ were extracted from a database registry in Ayatollah Taleghani Hospital, affiliated to Abadan University of Medical Sciences, which is a central hospital for COVID-19 diagnosis and treatment in the southwest of Khuzestan Province, Iran. The time frame of this study was from February 9, 2020, to December 20, 2020. During this period, 10,800 suspected cases with COVID-19 were referred to Ayatollah Taleghani Hospital's Ambulatory and Emergency Departments. Of those, 2,394 cases were introduced as confirmed COVID-19 by real-time polymerase chain reaction (RT-PCR) test. Only the hospitalized patients who were diagnosed with positive RT-PCR tests were included in our study (see Fig. 1). All the data were checked by two health information management experts (MSH and HK-A) and a third researcher (KHM) adjudicated any variance in interpretation between the two primary reviewers. For different interpretations and missing data, we contacted the physicians who completed the form and the patient or their family members to review and supplement data. Finally, all the collected data were entered into an Excel file.

\section{Outcome variable}

The outcome variable was deceased representing in-hospital mortality with COVID-19 and had a twofold distribution: "Yes" if the patient is deceased or "No" otherwise.

\section{Preprocessing}

Patients who were lower than 18 years of age were excluded. These patients should be included in the scope of pediatric exploration. Patients discharged from the emergency department were excluded because their outcomes were unknown. We obtained de-identified data from 1626 patients in the Abadan CoV registry database. 116 incomplete case records which had many missing data (more than $70 \%$ ) were excluded from the investigation. Also, the remaining missing values were imputed with the mean or mode of each variable. Noisy and abnormal values, errors, duplicates, and meaningless data were checked by two health information management (HIM) experts ( $\mathrm{MSH}$ and $\mathrm{HKH}$ ) in collaboration with two infectious diseases and hematology specialists. For different interpretations about data preprocessing, we contacted the corresponding physicians. The final sample size used in this analysis was 1,500 hospitalized patients who were over 18 years old. For detailed exclusions, a schematic of all the study inclusion criteria is shown in Fig. 1. 


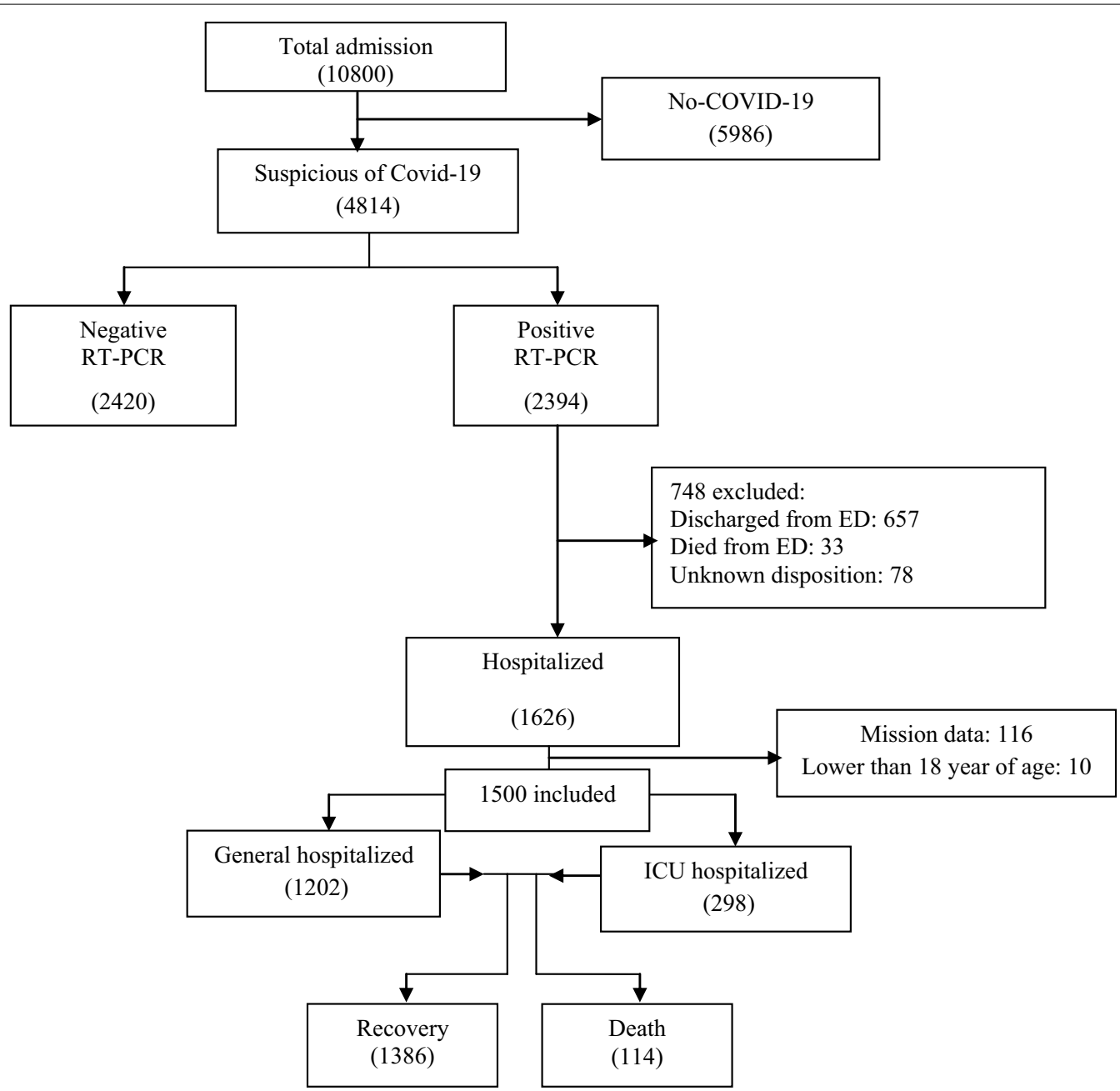

Fig.1 Flowchart describing patient selection

\section{Data balancing}

One of the key barriers to ML algorithms is the imbalanced data problem. This occurs when the classes are not categorized equally. In the selected dataset, the amount of data in outcome classes is significantly imbalanced and it contains more samples related to the alive class (1386 cases), while the death class is much smaller (only 114 cases). Accordingly, the trained models are often delivering prejudiced results towards overriding class and the ML models are much more possible to categorize new observations to the majority class. In this study, to handle the class imbalance, the synthetic minority over-sampling technique (SMOTE) method was used in the imbalanced-learn toolbox to balance the dataset (https://imbalanced-learn.org/stable/).

\section{Feature selection}

Feature selection is a technique commonly used in forecasting, pattern recognition, and classification modeling to lessen the dimensions and intricacy of the dataset by discarding irrelevant and redundant features. In this study, feature selection was performed to set up a model and order the input features according to their importance concerning the specific problem or target classes [20, 24]. Many feature selection methods have been suggested to select suitable features for predictive models, including Information GainRatio Attribute evaluation (GA), Forward Elimination, Backward Elimination, and One Rule Attribute Evaluation (ORAE) [25]. In this study, the GA method in Waikato Environment for Knowledge Analysis (Weka) (v3.9.2) software was used to select the 
features. This method measures the importance of features with respect to target class on the basis of gain ratio $[25,26]$. It can be calculated by the following formula, on the various evaluation measures that are available in WEKA [32, 33]. In tenfold cross-validation process, the original samples are randomly partitioned into 10 sub-

GainR $($ Class, feature $)=(\mathrm{H}($ Class $)-H($ Class $\mid$ feature $)) / H($ feature $)$

where Class $=$ Binary outcome (DN/absence of nephropathy), features $=$ Evaluated parameter $(B P$, GFR, HbA1c, UACR, etc.), also seen as risk factor.

\section{Model development}

The predictive classifier models were developed for accurately predicting COVID-19 mortality. In the modeling stage, to select the appropriate ML algorithms, the related studies in this field were reviewed $[10,11,15,18$, 23, 27-31] along with considering the type and quality of the selected dataset. To construct the mortality prediction model, we applied seven ML algorithms including the 548 decision tree, random forest (RF), k-nearest neighborhood (k-NN), multi-layer perceptron (MLP), Naïve Bayes (NB), eXtreme gradient boosting (XGBoost), and logistic regression (LR). The SPSS software (version 23) was used to analyze the data. Finally, the algorithms were implemented using Weka (v3.9.2) software to analyze and calculate the curves and criteria, and draw the confusion.

\section{Cross-validation}

We apply WEKA's EXPLORER module to determine the optimal hyperparameters for all models used. The hyperparameters selected were those that attained the best performance values. A tenfold cross-validation process system was used for evaluating the performance and general error of whole classification models. By using WEKA's EXPERIMENTER module, running all models ten times, and using repeated tenfold cross-validation, to ease comparing of the predictive performance based

Table 1 Confusion matrix

\begin{tabular}{lll}
\hline Output & Predicted values & \\
\cline { 2 - 3 } & Death $(+)$ & Survive (-) \\
\hline Actual value & & \\
Death (+) & TP & FN \\
Survive (-) & FP & TN
\end{tabular}

True Positive $=$ the number of cases that are truly classified as positive by the algorithm

False Positive $=$ the number of cases that are falsely classified as positive by the algorithm

False Negative $=$ the number of cases that are falsely classified as negative by the algorithm

True Negative $=$ the number of cases that are truly classified as negative by the algorithm samples of about equal size. One of the 10 sub-samples was applied as the validation dataset for testing the models, and the remaining 9 sub-samples were applied as training datasets. The cross-validation method was then repeated 10 times with one of the 10 sub-samples applied sequentially for each validation. Finally, the validation results from ten experimental models are then mixed to render the performance metrics (sensitivity, specificity, accuracy, precision, and ROC) derived from testing only $[34,35]$. In the other hand, the results of the performance metrics are computed as an average of these ten runs [36-39]. In general, stratified tenfold cross-validation is proposed for estimation accuracy as a result of its relatively low-level bias and variety.

It should be noted that the ten-fold cross-validation is a widely applied and preferred validation technique in machine learning and data mining due to differing from the conventional split instance method. This method assisted to reduce the deviation in prediction error; increases the use of data for both training and validation, without overfitting or overlap between the test and validation data; and protectors against experiment theory proposed by arbitrarily split data [40].

\section{Model evaluation}

Model performance evaluation is a fundamental part of building an effective ML model. The predictive models were evaluated using confusion matrix performance metrics (Table 1). In order to evaluate the predictive models, we applied some evaluation measures metrics including accuracy, specificity, precision, sensitivity, and receiver operating characteristic (ROC) chart criteria to measure the model's performance. Finally, all these evaluation criteria were compared in terms of the performance to get the best model for predicting the COVID-19 moralities (Table 2).

Table 2 The performance evaluation measures

\begin{tabular}{ll}
\hline Performance criteria & Calculation \\
\hline Accuracy & $(\mathrm{TP}+\mathrm{TN}) /(\mathrm{TP}+\mathrm{TN}+\mathrm{FP}+\mathrm{FN})$ \\
Precision & $\mathrm{TP} /(\mathrm{TP}+\mathrm{FP})$ \\
Sensitivity/ Recall & $\mathrm{TP} /(\mathrm{TP}+\mathrm{FN})$ \\
Specificity & $\mathrm{TN} /(\mathrm{TN}+\mathrm{FP})$ \\
\hline
\end{tabular}

True Positive (TP), True Negative (TN), False Positive (FP), False Negative (FN) 


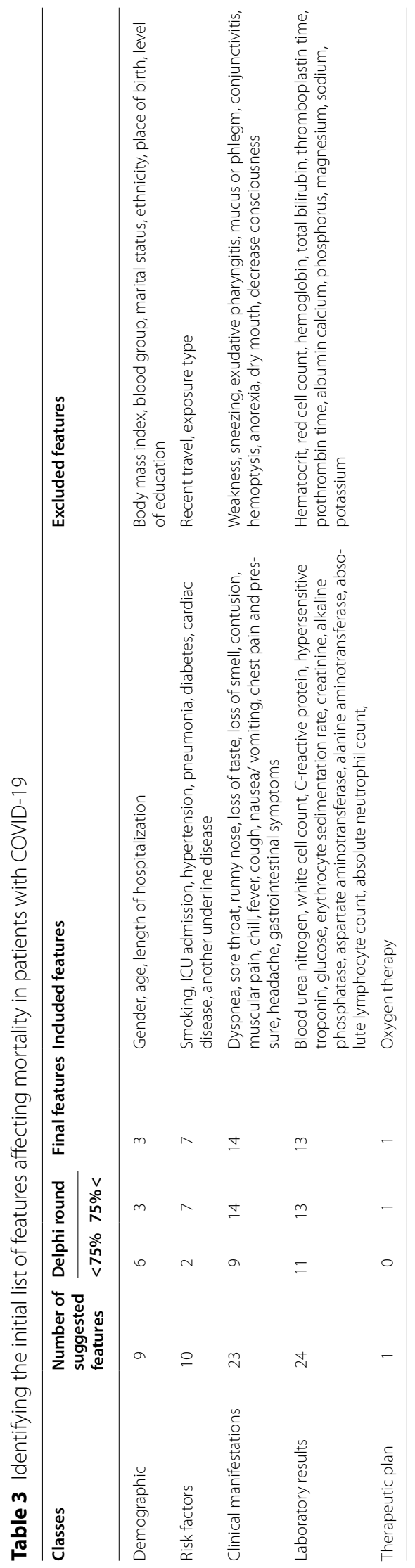




\section{Ethical considerations}

This study was approved by Ethical Committee Board, Abadan University of Medical Sciences (code: IR.ABADANUMS.REC.1400.008). In order to protect the privacy and confidentiality of the patients, we concealed the unique identifying information of all the patients in the process of data collection.

\section{Results}

The results of the six stages of the study are presented below:

\section{Feature identification and selection}

After conducting a comprehensive literature review along with a two-round Delphi survey, 54 clinical features were identified as probable predictors for determining the mortality risk of COVID-19 patients. In the next step, the degree of each factor in predicting COVID-19 hospitalized mortality based on GA method evaluation was calculated. Based on this method of 54 clinical features that remained until this step, 16 features were excluded from the study and 38 predictors were chosen as the input for the ML algorithms (Table 3). These features were divided into six categories, including demographics, risk factors, clinical manifestations, laboratory tests, and therapeutic plans.

In the selected feature list, dyspnea and platelet count with the correlation coefficient of 0.5532 and 0.0210 , respectively, gained the highest and lowest importance for predicting the COVID-19 mortality (Table 4).

\section{Patient selection}

We obtained data from 1626 patients in the Abadan $\mathrm{CoV}$ registry database. One hundred and sixteen incomplete records which had many missing data (more than $70 \%)$ were excluded from the analysis. The final sample size used in this analysis was 1500 adult patients (over 18 years old) who were hospitalized in the hospital. For the detailed exclusions, see Fig. 1. It should be noted that Fig. 1 was designed and created by the authors according to the steps taken to select the patients.

\section{Participants' characteristics}

After applying the exclusion criteria and quantitative analysis of case records, the number of 1500 hospitalized COVID-19 patients met eligibility. 836 patients (55.74\%) were male and $664(44.26 \%)$ were women, and the median age of the participants was 57.25 years old (interquartile 18-100). In total, 298 (19.87\%) were hospitalized in ICU and $1202(80.13 \%)$ were in general wards. Of these, 1386 (92.4\%) recovered and 114 (7.6\%) deceased (Table 5).
Developing and evaluating models

After selecting the best feature subset, we used various ML algorithms to build a predictive model. In this research, seven ML algorithms, including J48, MLP, XGBoost, RL, k-NN, RF, and NB, were trained for developing COVID-19 mortality prediction models. Then, the performance of each developed model was evaluated using sensitivity, specificity, accuracy, precision, and ROC of the performance metrics (Table 6). As shown in this table, the RF algorithm reaching $90.70 \%$ sensitivity, 95.10\% specificity, $95.03 \%$ accuracy, $94.23 \%$ precision, and ROC value of $99.02 \%$ yielded better capability in predicting COVID-19 in-hospital mortality than other ML algorithms. Figure 2 depicted the performance metrics of the selected ML algorithms.

The results of comparing the area under the ROC curve for the selected ML algorithms are shown in Fig. 3.

Also, based on the ROC, the Naïve Bayes algorithm attained the worst performance with the sensitivity of $90.44 \%$, specificity of $84.31 \%$, accuracy of $87.47 \%$, precision of $81.32 \%$, and ROC of $92.05 \%$.

\section{Discussion}

The current study aimed to retrospectively develop and validate ML models based on the most relevant features in determining the risk of COVID-19 mortality derived from extensive literature review coupled with a two-round Delphi survey. For this aim, the J48 decision tree, RF, k-NN, MLP, NB, XGBoost, and LR models were developed using a dataset of laboratory-confirmed COVID-19 hospitalized patients. The experimental results showed that RF had the best performance among the other seven ML techniques with the accuracy of $95.03 \%$, sensitivity of $90.70 \%$, precision of $94.23 \%$, specificity of $95.10 \%$, and ROC around $99.02 \%$. Our results showed that RF, XGBoost, KNN, and MLP models have a good prediction performance, the ROC is all above $96.49 \%$, and their diagnostic efficiency is better than the LR model trained using the same parameters.

Different studies have been evaluating the application of ML techniques in predicting mortality in the patients with COVID-19. Yadaw et al. [30] assessed the performance of four ML algorithms including $L R, R F$, SVM, and XGBoost using a dataset $(n=3841)$ for predicting COVID-19 mortality. The model developed with XGBoost happened to be the best model among all the models developed in terms of AUC with $0.91 \%$. In another study [23] a retrospective analysis on the data of 2520 COVID-19 hospitalized patients was conducted. Results of this study showed the model developed by the neural network (NN) yielded better performance and was the best model in terms of AUC with $0.9760 \%$ in predicting COVID-19 patient's physiological deterioration and 
Table 4 Features affecting predicting mortality in patients with COVID-19

\begin{tabular}{|c|c|c|c|c|c|}
\hline Row & Features name & $\begin{array}{l}\text { Degree of } \\
\text { importance }\end{array}$ & Row & Features name & $\begin{array}{l}\text { Degree of } \\
\text { importance }\end{array}$ \\
\hline 1 & Dyspnea & 0.5532 & 21 & Chest pain and pressure & 0.2256 \\
\hline 2 & ICU admission & 0.5409 & 22 & Absolute neutrophil count & 0.2123 \\
\hline 3 & Oxygen therapy & 0.3789 & 23 & Headache & 0.1992 \\
\hline 4 & Age & 0.3207 & 24 & Gender & 0.186 \\
\hline 5 & Fever & 0.3142 & 25 & Gastrointestinal symptoms & 0.1802 \\
\hline 6 & Cough & 0.3072 & 26 & White cell count & 0.1702 \\
\hline 7 & Loss of taste & 0.2944 & 27 & C-reactive protein & 0.1574 \\
\hline 8 & Loss of smell & 0.2923 & 28 & Hypersensitive troponin & 0.1428 \\
\hline 9 & Hypertension & 0.2768 & 29 & Pneumonia & 0.1066 \\
\hline 10 & Contusion & 0.2744 & 30 & Glucose & 0.0906 \\
\hline 11 & Muscular Pain & 0.2731 & 31 & Erythrocyte sedimentation rate & 0.0826 \\
\hline 12 & Chill & 0.2537 & 32 & Creatinine & 0.0716 \\
\hline 13 & Runny noise & 0.2532 & 33 & Alkaline phosphatase & 0.0678 \\
\hline 14 & Blood urea nitrogen & 0.2524 & 34 & Length of hospitalization & 0.0626 \\
\hline 15 & Diabetes & 0.2506 & 35 & Aspartate aminotransferase & 0.0445 \\
\hline 16 & Sore throat & 0.25 & 36 & Smoking & 0.0427 \\
\hline 17 & Absolute lymphocyte count & 0.2339 & 37 & Alanine aminotransferase & 0.0319 \\
\hline 18 & Nausea/vomiting & 0.2301 & 38 & Platelet count & 0.0210 \\
\hline 19 & Other under line disease & 0.2282 & 39 & & \\
\hline 20 & Cardiac disease & 0.2274 & & & \\
\hline
\end{tabular}

death among other models developed by logistic regression (LR), SVM, and gradient boosted decision tree. Vaid et al. [41] in their study analyzed data of 4029 confirmed COVID-19 patients from EHRs of five hospitals, and logistic regression with L1 regularization (LASSO) and MLP models was developed via local data and combined data. The federated MLP model (AUC-ROCs of $0.822 \%$ ) for predicting COVID-19 related mortality and disease severity outperformed the federated LASSO regression model. Other study conducted [42] four ML techniques were trained based on 10,237 patients' data and, finally, SVM with the sensitivity of $90.7 \%$, specificity of $91.4 \%$, and ROC of $0.963 \%$ had the best performance. Moulaei et al. [31] also predicted the mortality of Covid-19 patients based on data mining techniques and concluded that based on ROC (1.00), precision (99.74\%), accuracy (99.23\%), specificity (99.84\%) and sensitivity (98.25\%), RF was the best model in predicting mortality. After, the RF, KNN5, MLP, and J48 were the best models, respectively [31]

In the current study, some features such as dyspnea, ICU admission, oxygen therapy (intubation), age, fever, and cough were of the highest importance; on the other hand, alcohol/addiction, platelet count, alanine aminotransferase (ALT), and smoking were of the lowest importance in predicting COVID-19 mortality. However, from the physicians' point of view, awareness of these factors may be crucial for the success of drug therapy and mortality prediction. But in ML techniques, many of these factors can be ignored from analysis and mortality can be predicted with fewer factors.

Several studies have also reported some important clinical features(predictors) for COVID-19 patient mortality by leveraging a feature analysis technique. The selected features are used as inputs for developing MLbased models for severity, deterioration, and mortality of COVID-19 patient risk analysis. The strongest predictive features included basic data such as age (aged) [11, $17,28,30,43-46]$, gender (male) [10, 11, 18, 27, 29, 44, 46], BMI (high) [15-17], type of patient encounter (inpatient vs. outpatient) [11, 23, 27, 29], occupation (related to healthcare) $[17,23,29,30]$, clinical symptoms include dyspnea $[15,16,23,30,31,44,47]$, low consciousness $[11,17,18,28]$, dry cough $[15,17,18,23,27,28,44]$ fever $[11,17,18,43-45,47]$, para-clinical indicators consisting of spo2 (decreased) [16, 18, 29, 45, 47], lymphocyte count (low) [10, 23, 27-29], platelet count (low) [16, 27$29,47]$, leukocyte count (raised) $[15,16,27,28,30,44]$, neutrophil count (raised) [15, 23, 27, 28, 30, 43, 45], CRP (increased) $[15,29,30,45]$, D dimer (increased) [10, 30, 45], ALT and/or AST (raised) [16, 27, 28, 30, 47], cardiac troponin (increased) [23, 28, 29, 43], and LDH (elevated) $[17,27,28,48]$, and comorbidity conditions associated with poor prognosis including hypertension [28-30, 
Table 5 Descriptive statistics of the Features

\begin{tabular}{|c|c|c|}
\hline Features (quantitative) & Range & Mean (SD) \\
\hline Age (year) & $18-100$ & $57.25(17.8)$ \\
\hline Leng of hospitalization & $1-32$ & $61.89(13.25)$ \\
\hline Creatinine (mg/dL) & $0.1-17.9$ & $51.39(14.4)$ \\
\hline White-cell count & $1300-63,000$ & $82.34(4897.4)$ \\
\hline Platelet count & $108,000-691,000$ & $66.2(38.1)$ \\
\hline Absolute lymphocyte count & $2-95$ & $23.74(11.8)$ \\
\hline Absolute neutrophil count & $8-98$ & $74.52(12.3)$ \\
\hline Blood urea nitrogen & $0.5-251$ & $42.52(31.7)$ \\
\hline Aspartate aminotransferase & $3.8-924$ & $44.45(53.5)$ \\
\hline Alanine aminotransferase & $2-672$ & $38.29(41.6)$ \\
\hline Glucose & 18-994 & $36.09(74.2)$ \\
\hline Lactate dehydrogenase & $4.6-6973$ & $55.68(339.0)$ \\
\hline Prothrombin time & $0.9-46.8$ & $42.82(23.9)$ \\
\hline Alkaline phosphatase & $9.6-2846$ & $21.12(39.2)$ \\
\hline Erythrocyte sedimentation rate & $2-258$ & $40.65(28.8)$ \\
\hline Features (qualitative) & Values & Frequencies \\
\hline Gender & Male, Female & 836,664 \\
\hline Cough & Yes, No & 736,764 \\
\hline Contusion & Yes, No & 363,1137 \\
\hline Nausea/vomiting & Yes, No & 802,848 \\
\hline Headache & Yes, No & 899,601 \\
\hline Gastrointestinal symptoms & Yes, No & 976,524 \\
\hline Muscular pain & Yes, No & 1021,479 \\
\hline Chill & Yes, No & 878,622 \\
\hline Fever & Yes, No & 728,772 \\
\hline Pneumonia & Yes, No & 1044,456 \\
\hline Oxygen therapy & Yes, No & 1053,447 \\
\hline Dyspnea & Yes, No & 1078,422 \\
\hline Loss of taste & Yes, No & 272,1228 \\
\hline Loss of smell & Yes, No & 1195,305 \\
\hline Runny Noise & Yes, No & 637,863 \\
\hline Sore throat & Yes, No & 444,1056 \\
\hline Other underline disease & Yes, No & 360,1140 \\
\hline Cardiac disease & Yes, No & 118,1382 \\
\hline Hypertension & Yes, No & 395,1105 \\
\hline Diabetes & Yes, No & 268,1232 \\
\hline Smoking & Yes, No & 141,1359 \\
\hline Alcohol addiction & Yes, No & 26,1474 \\
\hline C-reactive protein & Positive, Negative & 1163,337 \\
\hline Oxygen therapy & Yes, No & 363,1137 \\
\hline ICU admission & Yes, No & 298,1202 \\
\hline Deceased & Yes, No & 114,1386 \\
\hline
\end{tabular}

44-46], lung disease including chronic obstructive lung disease $[11,16,27,28]$, asthma [16, 18], cardiovascular disease [28-30, 43, 45, 47], cancer [11, 44, 47], pneumonia $[11,17,46-48]$, and chronic renal disease $[11,15,17$, $18,46]$. On the other hand, sore throat $[11,27,28,30]$, myalgia and malaise [11, 29, 30], diarrhea and GI symptoms [23, 44, 45], and headache [11, 17, 47] for clinical manifestation and hemoglobin count $[11,15,45,47,48]$ as well as mean cell volume (MCV) $[16,17,28,44]$ and 
Table 6 Performance evaluation of the selected ML algorithms for COVID-19 death prediction

\begin{tabular}{llllcc}
\hline Algorithms & Sensitivity (\%) & Specificity (\%) & Accuracy (\%) & Precision (\%) & ROC (\%) \\
\hline Random forest & 90.70 & 95.10 & 95.03 & 94.23 & 99.02 \\
XGBoost & 90.89 & 95.01 & 94.25 & 92.43 & 98.18 \\
KNN & 97.38 & 82.15 & 89.56 & 80.11 & 97.19 \\
MLP & 90.81 & 91.07 & 91.25 & 83.94 & 96.49 \\
Logistic regression & 91.45 & 84.47 & 91.23 & 89.97 & 94.22 \\
J48 decision tree & 87.77 & 94.47 & 92.17 & 81.32 & 92.19 \\
Naîve Bayes & 90.44 & 84.31 & 87.47 & &
\end{tabular}

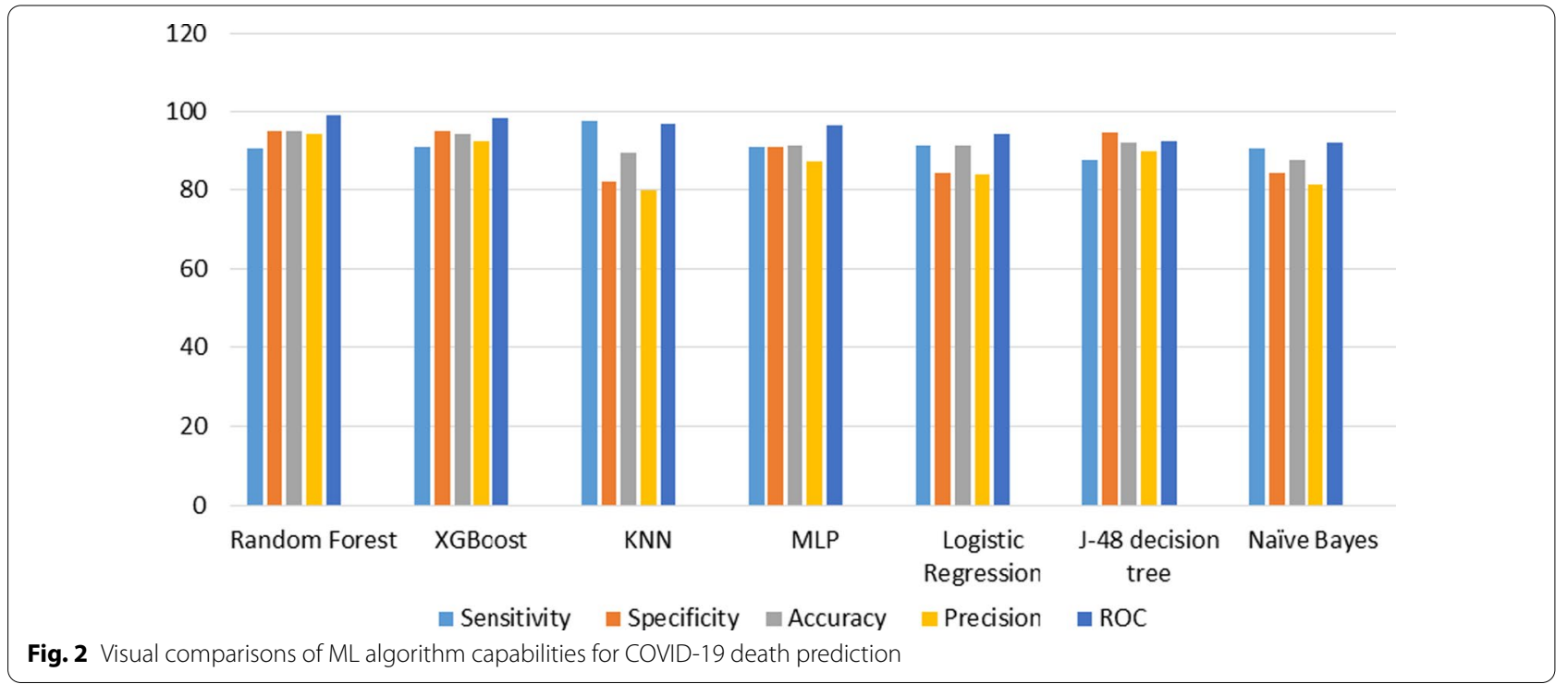

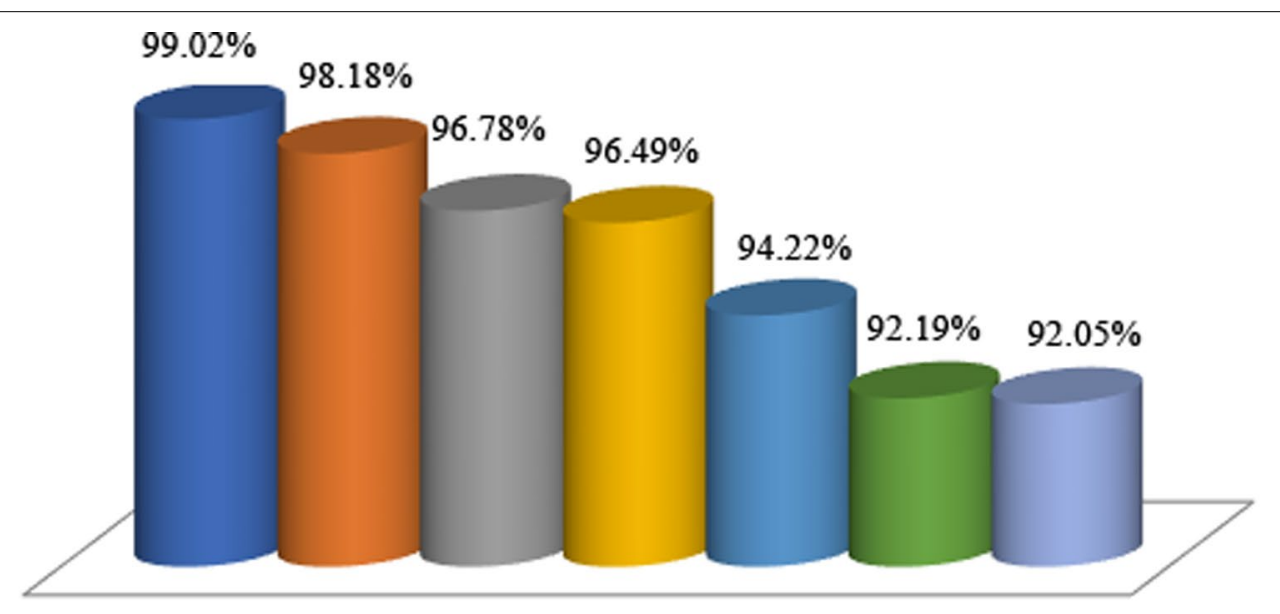

nandom Forest $\equiv$ XGBoost $\equiv \mathrm{KNN} \equiv \mathrm{MLP} \equiv$ Logistic regression $\equiv \mathrm{J} 48 \equiv$ Naïve Bayes

Fig. 3 ROC chart of selected ML algorithms 
hematocrit rate [18, 27-29] for the laboratory findings have the least importance for predicting.

Finally, ML can be of great use for the clinicians involved in treating the patients with COVID-19. The proposed algorithms can predict the mortality of the patients with optimum ROC, accuracy, precision, sensitivity, and specificity rates. This prediction can lead to the optimal use of hospital resources in treating the patients with more critical conditions and assisting in providing more qualitative care and reducing medical errors due to fatigue and long working hours in the ICU. Designing a valid predictive model may improve the quality of care and increase the survival rate of the patients. Therefore, predictive models for mortality risk analysis can greatly contribute to identifying high-risk patients and adopting the most effective assistive and treatment care plans. This could lead to decreasing ambiguity by offering quantitative, objective, and evidence-based models for risk stratification, prediction, and eventually episode of the care plan. It offers a better strategy for clinicians to lessen the complications and improve the likelihood of patient survival.

\section{Conclusion}

In this study, we created and evaluated ML-based prediction models for in-hospital mortality using the most important clinical features(38 predictors). The RF model performed best on classification accuracy among the other four ML algorithms. The proposed model can be suitably used for predicting the mortality risk of hospitalized COVID-19 patients and maximizing the use of restricted hospital resources. This model could automatically identify high-risk patients as early as the time of admission or during hospitalization. In conclusion, the use of ML algorithms in combination with qualitative and comprehensive hospital databases such as patient registries can enable timely and accurate mortality risk classification of COVID-19 patients. In the future, the performance of our model will be enhanced if we test more classification techniques at larger, multicenter, and qualitative datasets.

\section{Limitations}

Our work had several limitations that must be considered. First, this was a retrospective study design with the documented data that were irregular or imbalanced; thus, we balanced them by eliminating noise and inadequate records as much as possible from the dataset. To solve the imbalanced dataset problem, in which the number of records related to the dead class was significantly lower than the recovery or alive (144 vs 1386), different criteria were considered to measure the performance of each ML algorithm. Also, by using the SMOTE, the bias was minimized via class balancing. Another limitation was that it was conducted in a single-center registry database, which may limit the generalizability of the developed models. However, the ABADANUMS CoV registry is a database collected at a designated hospital in Abadan city that delivers special healthcare services to COVID19 patients. Nonetheless, we will use multi-center data to perform the external validation of the proposed model for enhancing the widespread prediction. Other features concerning the lung CT or radiology images could have been included. However, consistent with the purpose of the current research, considering only the routine clinical features of the patients while being admitted would suffice. Although the constraint of using data at admission inspires the usage of the model in patient triage, events that happened during a patient hospitalization period may drive their clinical course ahead of the previous likelihood, which cannot be apprehended by routine admission features. We believed a real-time or incessantly updating modeling method would be better matched for this as a future direction. Furthermore, we do not have information about the time span from symptom beginning to admission, which might have had an influence on the features that we sampled on hospital admission. Thus, the dynamic variations of some significant features must be followed up to better and timely recognize patients at higher risks of poor outcomes.

Finally, in the present study, patients who were less than 18 years of age and patients discharged from the emergency department were excluded from the study. If these people were included in the study, different results might have been obtained.

\section{Abbreviations \\ ML: Machine learning; RF: Random forest; k-NN: K-nearest neighborhood; MLP. Multi-layer Perceptron; NB: Naïve Bayes; ROC: Receiver operating characteristic; WHO: World Health Organization; Al: Artificial Intelligence; RT-PCR: Real-time polymerase chain reaction; TP: True Positive; TN: True Negative; FP: False Posi- tive; FN: False Negative; WEKA: Waikato Environment for Knowledge Analysis; XGBoost: EXtreme gradient boosting; NN: Neural network; LR: Logistic regres- sion; LASSO: Logistic regression with L1 regularization; MCV: Mean cell volume; SMOTE: Synthetic minority over-sampling technique.}

\section{Acknowledgements}

We thank the Research Deputy of the Abadan University of Medical Sciences for financially supporting this project. We also would like to thank all experts who participated in this study.

\section{Authors' contributions}

KHM, MSH: Conceptualization; Data gathering; Formal analysis; Investigation; Project administration; Resources; Supervision; Roles/Writing —original draft. ZM, HK: Conceptualization; Formal analysis; Investigation; Roles/Writing-original draft; Funding acquisition; Methodology; Writing-review and editing. KHM, MSH, HK: Conceptualization; Investigation; Methodology; Validation; Writing — review and editing. All authors read and approved the final manuscript.

Funding

There was no funding for this research project. 


\section{Availability of data and materials}

All data generated and analyzed during the current study are not publicly available but are available from the corresponding author on reasonable request and the Abadan University of Medical Sciences' approval.

\section{Declarations}

\section{Ethics approval and consent to participate}

This article is extracted from a research project supported by the Abadan University of Medical Sciences (IR.ABADANUMS.REC.1399.143). All methods of the present study were performed in accordance with the relevant guidelines and regulations of the ethical committee of Abadan Faculty of Medical Sciences. Participation was voluntary, the consent was verbal, but all participants responded via email or text message to approve their participation. Participants had the right to withdraw from the study at any time without prejudice.

\section{Consent for publication}

Not applicable.

\section{Competing interests}

The authors declare that they have no competing interests.

\section{Author details}

${ }^{1}$ Medical Informatics Research Center, Institute for Futures Studies in Health, Kerman University of Medical Sciences, Kerman, Iran. ${ }^{2}$ Department of Health Information Technology, School of Paramedical, Ilam University of Medical Sciences, Ilam, Iran. ${ }^{3}$ Department of Health Information Management, School of Health Management and Information Sciences, Kerman University of Medical Sciences, Kerman, Iran. ${ }^{4}$ Department of Health Information Technology, Abadan University of Medical Sciences, Abadan, Iran. ${ }^{5}$ Student Research Committee, Abadan University of Medical Sciences, Abadan, Iran.

Received: 26 May 2021 Accepted: 28 December 2021

Published online: 04 January 2022

\section{References}

1. Kazemi-Arpanahi H, Moulaei K, Shanbehzadeh M. Design and development of a web-based registry for Coronavirus (COVID-19) disease. Med J Islam Repub Iran. 2020;34:68-68.

2. Peeri NC, Shrestha N, Rahman MS, Zaki R, Tan Z, Bibi S, Baghbanzadeh M, Aghamohammadi N, Zhang W, Haque U. The SARS, MERS and novel coronavirus (COVID-19) epidemics, the newest and biggest global health threats: what lessons have we learned? Int J Epidemiol. 2020:2020:1-10.

3. Shi H, Han X, Jiang N, Cao Y, Alwalid O, Gu J, Fan Y, Zheng C. Radiological findings from 81 patients with COVID-19 pneumonia in Wuhan, China: a descriptive study. Lancet Infect Dis. 2020;20:425-34.

4. Liu Y, Wang Z, Ren J, Tian Y, Zhou M, Zhou T, Ye K, Zhao Y, Qiu Y, Li J. A COVID-19 risk assessment decision support system for general practitioners: design and development study. J Med Internet Res. 2020;22(6): e19786.

5. Alom MZ, Rahman M, Nasrin MS, Taha TM, Asari VK: COVID_MTNet: COVID-19 detection with multi-task deep learning approaches. 2020. arXiv preprint arXiv:2004.03747

6. Bansal A, Padappayil RP, Garg C, Singal A, Gupta M, Klein A. Utility of artificial intelligence amidst the COVID 19 pandemic: a review. J Med Syst. 2020:44(9):156

7. Lai C-C, Shih T-P, Ko W-C, Tang H-J, Hsueh P-R. Severe acute respiratory syndrome coronavirus 2 (SARS-CoV-2) and coronavirus disease-2019 (COVID-19): The epidemic and the challenges. Int J Antimicrob Agents. 2020;55(3): 105924.

8. Hussain A, Bhowmik B, do Vale Moreira NC. COVID-19 and diabetes: knowledge in progress. Diabetes Res Clin Practice. 2020;162: 108142.

9. Moujaess E, Kourie HR, Ghosn M. Cancer patients and research during COVID-19 pandemic: a systematic review of current evidence. Criti Rev Oncol/Hematol. 2020;150: 102972
10. Zhao Z, Chen A, Hou W, Graham JM, Li H, Richman PS, Thode HC, Singer AJ, Duong TQ. Prediction model and risk scores of ICU admission and mortality in COVID-19. PLoS ONE. 2020;15(7): e0236618.

11. Hu H, Yao N, Qiu Y. Comparing rapid scoring systems in mortality prediction of critically ill patients with novel coronavirus disease. Acad Emerg Med. 2020;27(6):461-8.

12. Shanbehzadeh M, Orooji A, Kazemi-Arpanahi H. Comparing of data mining techniques for predicting in-hospital mortality among patients with covid-19. J Biostat Epidemiol. 2021;7(2):154-73.

13. Josephus BO, Nawir AH, Wijaya E, Moniaga JV, Ohyver M. Predict mortality in patients infected with COVID-19 virus based on observed characteristics of the patient using logistic regression. Procedia Comput Sci. 2021;179:871-7.

14. Karthikeyan A, Garg A, Vinod PK, Priyakumar UD. Machine learning based clinical decision support system for early COVID-19 mortality prediction. Front Public Health. 2021;9: 626697.

15. Ryan L, Lam C, Mataraso S, Allen A, Green-Saxena A, Pellegrini E, Hoffman J, Barton C, McCoy A, Das R. Mortality prediction model for the triage of COVID-19, pneumonia, and mechanically ventilated ICU patients: a retrospective study. Ann Med Surg. 2020:59:207-16.

16. Wynants L, Van Calster B, Collins GS, Riley RD, Heinze G, Schuit E, Bonten MM, Dahly DL, Damen JA, Debray TP. Prediction models for diagnosis and prognosis of covid-19: Systematic review and critical appraisal. BMJ. 2020;369:1328

17. Wu G, Yang P, Xie Y, Woodruff HC, Rao X, Guiot J, Frix A-N, Louis R, Moutschen M, Li J. Development of a clinical decision support system for severity risk prediction and triage of COVID-19 patients at hospital admission: an international multicentre study. Eur Respir J. 2020;56(2):2001104.

18. Yan L, Zhang H-T, Goncalves J, Xiao Y, Wang M, Guo Y, Sun C, Tang X, Jing L, Zhang M. An interpretable mortality prediction model for COVID-19 patients. Nature Mach Intell. 2020;2:283-8.

19. Malki Z, Atlam E-S, Hassanien AE, Dagnew G, Elhosseini MA. Gad I: Association between weather data and COVID-19 pandemic predicting mortality rate: machine learning approaches. Chaos Solitons Fractals. 2020;138: 110137.

20. Shanbehzadeh M, Nopour R, Kazemi-Arpanahi H. Comparison of four data mining algorithms for predicting colorectal cancer risk. J Adv Med Biomed Res. 2021;29(133):100-8.

21. Hernandez-Suarez DF, Ranka S, Kim Y, Latib A, Wiley J, Lopez-Candales A, Pinto DS, Gonzalez MC, Ramakrishna H, Sanina C. Machine-learningbased in-hospital mortality prediction for transcatheter mitral valve repair in the United States. Cardiovasc Revasc Med. 2021;22:22-8.

22. Shipe ME, Deppen SA, Farjah F, Grogan EL. Developing prediction models for clinical use using logistic regression: an overview. J Thorac Dis. 2019;11(Suppl 4):S574.

23. Gao Y, Cai G-Y, Fang W, Li H-Y, Wang S-Y, Chen L, Yu Y, Liu D, Xu S, Cui P-F. Machine learning based early warning system enables accurate mortality risk prediction for COVID-19. Nat Commun. 2020;11(1):1-10.

24. Novaković J. Toward optimal feature selection using ranking methods and classification algorithms. Yugosl J Oper Res. 2016;21(1):119-35.

25. Nhu V-H, Shirzadi A, Shahabi H, Singh SK, Al-Ansari N, Clague JJ, Jaafari A, Chen W, Miraki S, Dou J, et al. Shallow landslide susceptibility mapping: a comparison between logistic model tree, logistic regression, Naïve Bayes tree, artificial neural network, and support vector machine algorithms. Int J Environ Res Public Health. 2020;17(8):2749.

26. Rodriguez-Romero V, Bergstrom RF, Decker BS, Lahu G, Vakilynejad M, Bies RR. Prediction of nephropathy in type 2 diabetes: an analysis of the ACCORD trial applying machine learning techniques. Clin Transl Sci. 2019;12(5):519-28.

27. Booth AL, Abels E, McCaffrey P. Development of a prognostic model for mortality in COVID-19 infection using machine learning. Mod Pathol. 2021:34:522-31.

28. Das AK, Mishra S, Gopalan SS. Predicting CoVID-19 community mortality risk using machine learning and development of an online prognostic tool. PeerJ. 2020;8: e10083.

29. Pan P, Li Y, Xiao Y, Han B, Su L, Su M, Li Y, Zhang S, Jiang D, Chen X, et al. Prognostic assessment of COVID-19 in the intensive care unit by machine learning methods: model development and validation. J Med Internet Res. 2020;22: e23128. 
30. Yadaw AS, Li Y-C, Bose S, lyengar R, Bunyavanich S, Pandey G. Clinical features of COVID-19 mortality: development and validation of a clinical prediction model. Lancet Digit Health. 2020;2(10):e516-25.

31. Moulaei K, Ghasemian F, Bahaadinbeigy K, Ershad sarbi R, Mohamadi Taghiabad Z. Predicting mortality of COVID-19 patients based on data mining techniques. J Biomed Phys Eng. 2021;11:653-62.

32. Kate RJ, Perez RM, Mazumdar D, Pasupathy KS, Nilakantan V. Prediction and detection models for acute kidney injury in hospitalized older adults. BMC Med Inform Decis Mak. 2016:16(1):1-11.

33. Serviá L, Montserrat N, Badia M, Llompart-Pou JA, Barea-Mendoza JA, Chico-Fernández M, Sánchez-Casado M, Jiménez JM, Mayor DM, Trujillano J. Machine learning techniques for mortality prediction in critical traumatic patients: anatomic and physiologic variables from the RETRAUCl study. BMC Med Res Methodol. 2020;20(1):1-12.

34. Utami E, Raharjo S. Mortality prediction using data mining classification techniques in patients with hemorrhagic stroke. In: 2020 8th international conference on cyber and IT service management (CITSM): 2020. IEEE; 2020, p. 1-5.

35. Motwani M, Dey D, Berman DS, Germano G, Achenbach S, Al-Mallah MH, Andreini D, Budoff MJ, Cademartiri F, Callister TQ. TQJEhj: machine learning for prediction of all-cause mortality in patients with suspected coronary artery disease: a 5-year multicentre prospective registry analysis. Eur Heart J. 2017;38(7):500-7.

36. Motwani M, Dey D, Berman DS, Germano G, Achenbach S, Al-Mallah $\mathrm{MH}$, Andreini D, Budoff MJ, Cademartiri F, Callister TQ. Machine learning for prediction of all-cause mortality in patients with suspected coronary artery disease: a 5-year multicentre prospective registry analysis. Eur Heart J. 2017;38(7):500-7.

37. Molinaro AM, Simon R, Pfeiffer RM. Prediction error estimation: a comparison of resampling methods. Bioinformatics. 2005;21(15):3301-7.

38. Attwal KPS, Dhiman AS. Exploring data mining tool-Weka and using Weka to build and evaluate predictive models. Adv Appl Math Sci. 2020;19(6):451-69.

39. Asadabadi EB, Abdolmaleki P. Predictions of protein-protein interfaces within membrane protein complexes. Avicenna J Med Biotechnol. 2013;5(3):148-57.

40. Wu C-C, Yeh W-C, Hsu W-D, Islam MM, Nguyen PAA, Poly TN, Wang Y-C, Yang $\mathrm{H}-\mathrm{C}$, Li Y-CJ. Prediction of fatty liver disease using machine learning algorithms. Comput Methods Programs Biomed. 2019;170:23-9.

41. Vaid A, Jaladanki SK, Xu J, Teng S, Kumar A, Lee S, Somani S, Paranjpe I, De Freitas JK, Wanyan T. Federated learning of electronic health records improves mortality prediction in patients hospitalized with covid-19. medRxiv 2020

42. An C, Lim H, Kim D-W, Chang JH, Choi YJ, Kim SW. Machine learning prediction for mortality of patients diagnosed with COVID-19: a nationwide Korean cohort study. Sci Rep. 2020;10(1):1-11.

43. Allenbach Y, Saadoun D, Maalouf G, Vieira M, Hellio A, Boddaert J, Gros $H$, Salem JE, Resche Rigon M, Menyssa C. Development of a multivariate prediction model of intensive care unit transfer or death: A French prospective cohort study of hospitalized COVID-19 patients. PLOS ONE. 2020;15(10): e0240711.

44. Assaf D, Gutman Y, Neuman Y, Segal G, Amit S, Gefen-Halevi S, Shilo N, Epstein A, Mor-Cohen R, Biber A. Utilization of machine-learning models to accurately predict the risk for critical COVID-19. Internal Emerg Med. 2020;15(8):1435-43.

45. Zhang Y, Xin Y, Li Q, Ma J, Li S, Lv X, Lv W. Empirical study of seven data mining algorithms on different characteristics of datasets for biomedical classification applications. Biomed Eng Online. 2017;16(1):125

46. Zhou $Y$, He Y, Yang H, Yu H, Wang T, Chen Z, Yao R, Liang Z. Exploiting an early warning Nomogram for predicting the risk of ICU admission in patients with COVID-19: a multi-center study in China. Scand J Trauma Resusc Emerg Med. 2020;28(1):1-13.

47. Chin V, Samia NI, Marchant R, Rosen O, loannidis JP, Tanner MA, Cripps S. A case study in model failure? COVID-19 daily deaths and ICU bed utilisation predictions in New York State. Eur J Epidemiol. 2020;35(8):733-42.

48. Agieb R. Machine learning models for the prediction the necessity of resorting to icu of covid-19 patients. Int J Adv Trends Comput Sci Eng. 2020;2020:6980-4.

\section{Publisher's Note}

Springer Nature remains neutral with regard to jurisdictional claims in published maps and institutional affiliations.
Ready to submit your research? Choose BMC and benefit from:

- fast, convenient online submission

- thorough peer review by experienced researchers in your field

- rapid publication on acceptance

- support for research data, including large and complex data types

- gold Open Access which fosters wider collaboration and increased citations

- maximum visibility for your research: over $100 \mathrm{M}$ website views per year

At BMC, research is always in progress.

Learn more biomedcentral.com/submissions 